\title{
Direct Quantification of Intraparticle Protein Diffusion in Chromatographic Media
}

\author{
Magnus Schröder, Eric von Lieres, and Jïrgen Hubbuch* \\ Institute of Biotechnology, Research Centre Jülich, 52425 Jülich, Germany \\ Received: August 2, 2005; In Final Form: November 17, 2005
}

\begin{abstract}
Diffusion coefficients of proteins in chromatographic media are important parameters for the rational design of stationary phases and purification schemes. In contrast to free diffusion, intraparticle diffusion is hindered by the porous structure of the media. Direct intraparticle diffusion analysis (IDA) is a novel approach for the determination of intraparticle protein diffusion coefficients. IDA is based on the evaluation of spatially and temporally resolved intraparticle concentration profiles. To prevent adsorption and to study diffusion only, the chromatographic media are investigated in underivatized form. With IDA, intraparticle concentration profiles are measured in a microcolumn by confocal laser scanning microscopy (CLSM). From this dynamic data, the diffusion coefficients are determined by parameter estimation, using a spheric diffusion model. The boundary condition is given by the measured protein concentration in the bulk phase. IDA is applied to determine intraparticle diffusion coefficients of seven different proteins in Sepharose 6 FF. The results show excellent congruence of experimental data and simulation results. Moreover, the determined diffusion coefficients lie well within the range of data published in the literature. Given that the material in question allows optical analysis, IDA is a general approach for studying protein diffusion in porous particles and is easily adapted to different proteins, solution conditions and stationary phases.
\end{abstract}

\section{Introduction}

Chromatographic separations are the method of choice for most protein purification applications using columns packed with porous particles. Consequently, a wide variety of mathematical models were developed in order to predict chromatographic performance. The simulation results rely, besides other parameters, on a precise knowledge of diffusion coefficients within the porous adsorbent.

It has been shown that pore diffusion is often the rate-limiting step in both affinity ${ }^{1}$ and ion exchange chromatography. ${ }^{2}$ Diffusion of macromolecules in porous structures is hindered in comparison to diffusion in free solution. This hindrance can be described as a function of solute size and geometric properties of the porous network or by an effective medium approach on the basis of the hydraulic permeability.

However, each stationary phase has different properties, which makes a priori prediction of intraparticle diffusion coefficients a difficult task. Thus, several experimental techniques were developed to measure diffusion coefficients in porous media in the past.

To obtain diffusion coefficients of proteins in porous media, columns packed with the respective media are used in conjunction with a chromatographic system and suitable detectors. The protein solution is injected either as a pulse $e^{3,4}$ or by a step change, ${ }^{5}$ and the protein concentration is measured at the column exit. The tank-in-series ${ }^{4}$ and continuum ${ }^{5}$ models, the moment, height equivalent to plate, or fast Fourier transform methods ${ }^{3}$ were used to calculate diffusion coefficients from the obtained chromatogram. Using such indirect methods, great care has to be taken to account for extra column dispersion.

A stirred cell apparatus has recently been used to measure diffusion coefficients in agarose. ${ }^{6}$ A simple mass balance is

* Corresponding author: e-mail, j.hubbuch@fz-juelich.de; phone, +492461-61-6044; fax, +49-2461-61-3870. employed to obtain the diffusion coefficient of a protein, but experiments have a very long duration $(>100 \mathrm{~h})$, such that sensitive proteins may undergo conformational changes and the apparatus cannot utilize chromatographic particles.

Fluorescence recovery after photobleaching (FRAP) in agarose media is often used to determine the diffusion coefficient of macromolecules in porous media. ${ }^{7-10}$ Porous beads are mixed with fluorescently labeled proteins until equilibrium is reached. When a laser beam is focused with high intensity on a small region of the bead, the fluorescent molecules are bleached instantly. After that, an attenuated laser beam is used to monitor the influx of nonbleached fluorescently labeled proteins into the bleached area with a microscope. The diffusion coefficient is determined by fitting a model to the fluorescence recovery curve directly obtained from the images ${ }^{8-10}$ or fast Fourier transformed images. ${ }^{7}$ Even though this technique offers a fast and excellent way to determine diffusion coefficients under steady-state conditions, it cannot resolve dynamic conditions occurring due to concentration gradients in chromatographic systems. However, nondiffusional recovery of the fluorescence signal is possible due to reversible bleaching of dyes, and an influence of the attached fluorescent marker on the intraparticle diffusion coefficient could lead to erroneous results.

Other approaches for the experimental determination of intraparticle diffusion coefficients apply holographic laser interferometry ${ }^{11,12}$ or electronic speckle interferometry. ${ }^{13}$ Due to changes in protein concentration inside the gel, a change in the local refractive index can be measured with interferometric methods. The obtained data are characteristic fringe patterns that are monitored with a CCD camera or a holographic plate. The digitized patterns are then treated with image analysis software together and model-based analyzed to convert the fringe patterns to information on local protein concentrations. These are subsequently used to obtain the protein diffusion coefficient. Interferometric techniques are applied for the 
analysis of non-cross-linked agarose. Hence, the matrix is different from the media, which are typically used for chromatographic separation. Furthermore, experiments for large molecules are time-consuming $(>12 \mathrm{~h})$.

Another method for the determination of diffusion coefficients is based on pulsed field gradient nuclear magnetic resonance (PFG-NMR) spectroscopy. ${ }^{14-16}$ The NMR system essentially detects the motion of molecules by magnetically tagging and monitoring them for short periods of time. To solely detect the motion of the protein and not the solvent, the system has to be completely deuterated or the protein must be fluorinated and fluorine PFG-NMR has to be used. With the PFG-NMR technique even opaque stationary phases can be analyzed, but the detector does not distinguish between the particle phase and the interparticle volume. Hence, the interparticle space has to be replaced with a nonaqueous solvent, to observe intraparticle diffusion.

In this paper, direct intraparticle diffusion analysis (IDA) is presented as a novel approach for the determination of intraparticle protein diffusion coefficients. IDA dynamically observes protein diffusion in porous particles, which are used for chromatography, in a packed microcolumn by means of confocal laser scanning microscopy. The experimental setup allows observation of diffusion under typical chromatographic operating conditions. The obtained data are analyzed utilizing a spheric diffusion model. Intraparticle diffusion coefficients are accurately determined by parameter estimation.

IDA is applied for diffusion analysis of seven proteins in underivatized Sepharose $6 \mathrm{FF}$, which is composed of crosslinked agarose and contains negligible amounts of charged residues. It is well documented, that nonspecific interactions between the proteins and the particle are largely avoided, due to the hydrophilic nature of the agarose backbone. Moreover, a moderate salt concentration is used in the present study, to screen for remaining interactions between proteins and the stationary phase.

\section{Materials and Methods}

Chemicals, Proteins, and Media. Unless otherwise noticed, all chemicals were obtained from Merck in analytical grade. All solutions were prepared in MilliQ water. Seven different proteins were analyzed in this study: bovine serum albumin (BSA, Sigma, A6003), chicken egg ovalbumin (OVA, Sigma, A5503), $\beta$-lactoglobulin (BLG, Sigma, L3908), iron free chicken egg ovotransferrin (OVT, Sigma, C0755), hen egg white lysozyme (LYS, Merck, 105281), porcine $\gamma$-globulin (GGB, Sigma, G2512), and bovine $\alpha$-chymotrypsinogen A (CTG, Worthington Biochemicals, LS005623). In addition, blue dextran (Sigma, D5751), BODIPY FL NHS Ester (Molecular Probes, D2184), and Atto-635 NHS Ester (Atto-Tec GmbH, A635-3) were used in column experiments. For diffusion experiments Sepharose 6 FF (GE Healthcare) was used as the stationary phase. The samples were donated by GE Healthcare. Sepharose $6 \mathrm{FF}$ is composed of $6 \%$ cross-linked agarose, with an average particle size of $90 \mu \mathrm{m}$ and a particle size distribution between 45 and $165 \mu \mathrm{m}$. SP Sepharose FF, the derivatized version of Sepharose $6 \mathrm{FF}$, has been reported with an average pore size of $24.7 \mathrm{~nm}^{17}$

Protein Labeling. The proteins were labeled with fluorescent amine-reactive Cy5 NHS ester (GE Healthcare) in $50 \mathrm{mM}$ phosphate buffer at $\mathrm{pH} 7.0$ for $1 \mathrm{~h}$. The Cy5 conjugate was purified from unreacted dye over a self-packed gel filtration column (XK16/20, GE Healthcare, $1.6 \mathrm{~cm}$ inner diameter, 16 cm column length). The column was filled with G-15 Sephadex

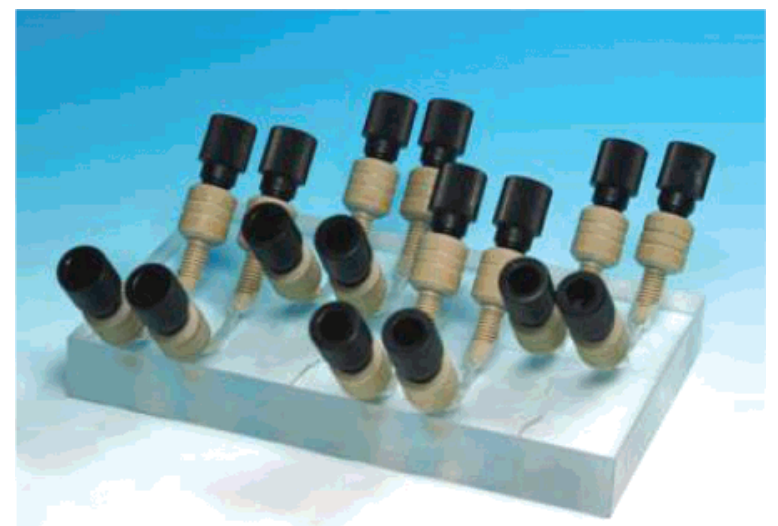

Figure 1. Plexiglas block with eight custom designed microcolumns.

(GE Healthcare) and equilibrated with $25 \mathrm{mM}$ phosphate buffer at pH 7.0 with $200 \mathrm{mM} \mathrm{NaCl}$ using an Äkta Basic 10 system (GE Healthcare). The degree of labeling was determined by UV-vis spectroscopy on a Cary 50 (Varian) using the molar extinction coefficient of $\mathrm{Cy} 5$ at $654 \mathrm{~nm}$ and the extinction coefficient of the proteins at $280 \mathrm{~nm}$ corrected for the absorption of Cy5 at $280 \mathrm{~nm}$. Unlabeled protein solutions were mixed with labeled ones to yield a total protein concentration of $3.5 \times 10^{-5}$ $\mathrm{M}$ and a molar dye-to-protein ratio (D/P) of 0.1 . The final protein solution buffer was composed of $25 \mathrm{mM}$ phosphate buffer at pH 7.0 with $200 \mathrm{mM} \mathrm{NaCl}$.

Fluorescence Signal Linearity Test. To test whether the chosen Cy5 concentrations were in the linear region for detection with the CLSM, $15 \mu \mathrm{L}$ of a $10 \%$ (w/v) slurry of Sepharose 6 FF was dispensed in wells of a 96-well plate with a glass bottom (Whatman, 7706-1365). A dilution series of BSA-Cy5 was set up, covering Cy5 concentrations between $3.02 \times 10^{-6}$ to 2.71 $\times 10^{-5} \mathrm{M}$ with a constant BSA concentration. One hundred microliters of the respective protein solution was added to the wells. After equilibrium was reached, a particle from each well was analyzed using the same microscope settings for every well. The conditions for the particle analysis were the same as outlined below. The average bulk and particle fluorescence signal intensities were calculated from the images by manual determination of a suitable region and calculation of the average signal intensity.

Interaction of Fluorescent Labels with the Stationary Phase. A Tricorn 5/200 column (GE Healthcare), packed with Sepharose $6 \mathrm{FF}$, was connected to a high-performance liquid chromatography (HPLC) system (Shimadzu LC10ADvp) with autoinjector (Shimadzu, SIL-ADvp) and diode array (SPDM10ADvp) detection for pulse injections of fluorescent dyes or labeled proteins. The column was equilibrated with phosphate buffer $(25 \mathrm{mM}, \mathrm{pH} 7.0,200 \mathrm{mM} \mathrm{NaCl})$ at a flow rate of 0.2 $\mathrm{mL} / \mathrm{min}$. Subsequently $20 \mu \mathrm{L}$ of solution, dissolved in the same buffer, was injected on the column and eluted isocratically.

Fluorescence Correlation Spectroscopy (FCS). Measurement of free diffusion coefficients of Cy5 labeled proteins were done with a FCS method essentially described in Böhmer et al. ${ }^{18}$ with a total protein concentration of $3.5 \times 10^{-5} \mathrm{M}$ in a phosphate buffer (pH 7.0, $25 \mathrm{mM}, 200 \mathrm{mM} \mathrm{NaCl}$ ) and a labeled protein concentration of $\sim 1 \mathrm{nM}$.

Experimental Setup for Intraparticle Diffusion Analysis. A custom designed microcolumn was used for the measurement of intraparticle concentration profiles. ${ }^{19}$ This microcolumn is integrated in a Plexiglas block (Figure 1).

In variation to Hubbuch et al., ${ }^{19}$ a union $1 / 16$ in. female/M6 male (GE Healthcare) was inserted into the external M6 adaptor 


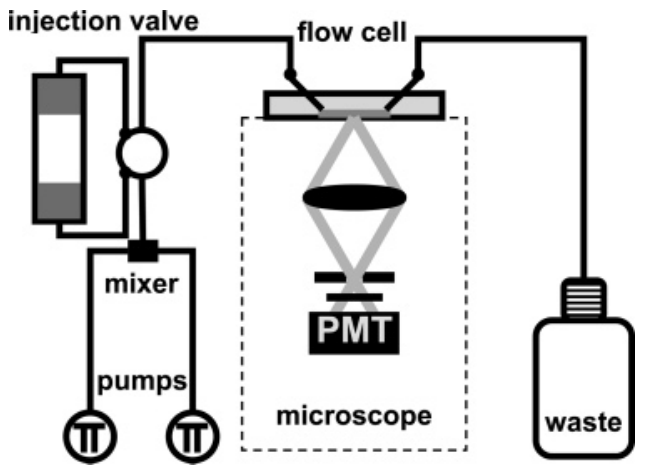

Figure 2. Experimental setup for intraparticle diffusion analysis using confocal laser scanning microscopy and a high-pressure gradient system: injection volume, $400 \mu \mathrm{L}$; flow rate, $0.1 \mathrm{~mL} / \mathrm{min}$; glass coverslip glued to the bottom of a flow cell; observed particle near the inlet of the packed bed; PMT, photomultiplier tube.

(P-653, Upchurch Scientific) of the flow cell. This setup was used to minimize dead volumes by directly connecting the flow cell to the HPLC. Furthermore, the dimension of the flow channel was changed to $2 \mathrm{~mm}$ inner diameter, $2 \mathrm{~mm}$ visible length, and $2 \mathrm{~mm}$ height. The flow cell was packed with slurried Sepharose 6 FF (GE Healthcare) particles in $1 \mathrm{M} \mathrm{NaCl}$, using a $1 \mathrm{~mL}$ syringe and avoiding introduction of air bubbles. The complete experimental setup is presented in Figure 2.

The pumps of the gradient high-pressure system were primed with either phosphate buffer or sodium hydroxide. The microcolumn was connected and equilibrated with phosphate buffer at a flow rate of $0.2 \mathrm{~mL} / \mathrm{min}$ for at least 100 column volumes, to ensure good packing. The protein solution was placed into a $10 \mathrm{~mL}$ superloop (GE Healthcare) and injected to the column with a seven-port injection valve (Rheodyne). Simultaneously with the start of injection, image acquisition was started on a prechosen particle in the packed bed, with settings described in the next section, and continued until completion of the experiment. After injection of the protein solution, the increase in signal intensity in the bulk and particle phase was monitored. An injection of $400 \mu \mathrm{L}$ of protein solution permitted complete saturation of the packed bed. After protein supply from the superloop was stopped after $400 \mu \mathrm{L}$, the diffusion of the protein out of the particle was also monitored with the confocal setup. This setup allows measurement of the spatial and temporal protein concentration profile in the particle and bulk phase.

All experiments were carried out at room temperature and a flow rate of $0.1 \mathrm{~mL} / \mathrm{min}$. After each experiment, the column was regenerated using 5 column volumes of $0.1 \mathrm{M} \mathrm{NaOH}$ followed by equilibration with 10 column volumes of phosphate buffer. All experiments were repeated three times.

Microscope Settings. Fluorescence intensity profiles were measured with an inverted Zeiss LSM 510 confocal laser scanning microscope using a water immersion $63 \times /$ NA1.2 C-Apochromat (Zeiss) objective and a HeNe laser (633 nm). The laser intensity was kept constant for all experiments. Only slight adjustments $( \pm 20 \mathrm{~V})$ of the photomultiplier (PMT) detector gain were necessary to account for signal attenuation effects from neighboring particles in the packed bed. The intensity profiles were measured every second with $300-500$ frames in total. All profiles were stored as eight-bit single scans with a resolution of $512 \times 512$ pixels representing an area of $146.2 \times 146.2 \mu \mathrm{m}$. The chosen time interval allowed monitoring of the diffusion both into and out of the particle in one run. Bleaching of employed Cy5 dye was not observed (data not shown). Before each individual run, the reflection mode of the microscope (excitation wavelength $543 \mathrm{~nm}$ with 80/20 filter and

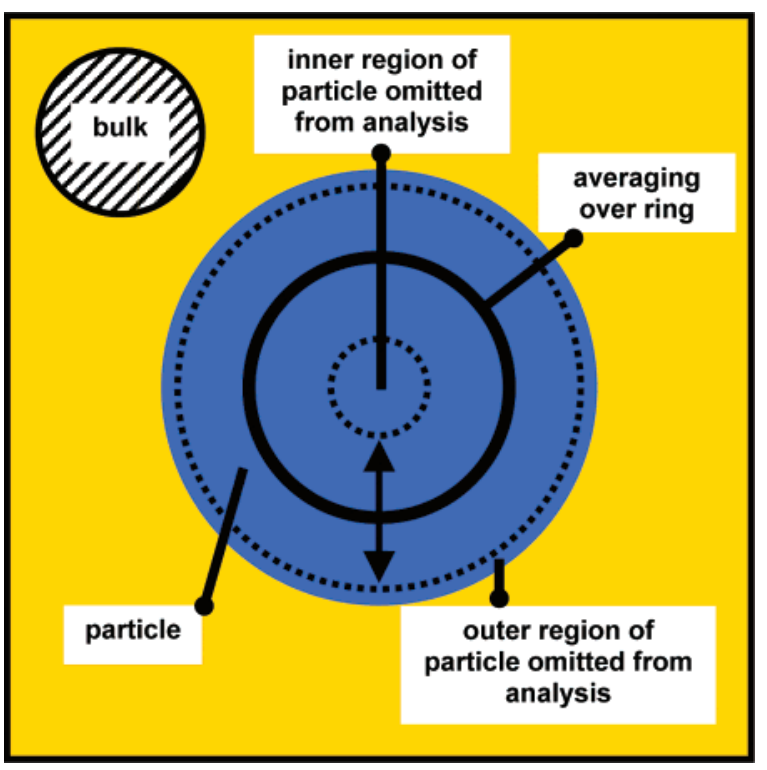

Figure 3. Schematic image from confocal laser scanning microscope. The bulk concentration (Figure 7) is determined from averaged signal intensities. The intraparticle concentration profile is determined from a manually chosen particle by averaging signal intensities at a given radius (black ring) over the whole azimuth of the particle. A $4.35 \mu \mathrm{m}$ region from the center and a $4.35 \mu \mathrm{m}$ region from the particle edge were omitted from data analysis.

no band- or long pass filter before the PMT) was used to verify that the focal plane went through the center of the particle. The image frame consisted of the particle of interest, other particles (focal plane not necessarily through particle center), and interstitial areas between the particles, from which the average bulk protein concentration was determined.

Data Preprocessing. The image processing software ImageJ (Version 1.33k, NIH, USA) was used for spatial averaging of both intraparticle and extraparticle data. The center of the analyzed particle as well as an extraparticle region with a distance of $10 \mu \mathrm{m}$ from any particle boundary was manually determined. Further data preprocessing, the model solution, and parameter estimation were implemented in MATLAB (Version 7.0.1, The MathWorks, USA).

For given radial positions, average values of the intensity data were generated, taking advantage of the spherical symmetry of the system (Figure 3). Figure 4a shows a typical example of this averaging procedure. The averaging of signal intensities over the particle azimuth at given points in time results in smoothed concentration profiles (Figure 4b). In comparison with concentration profiles over single radii with fixed azimuth, the smoothing procedure yields data which is much more suitable for further processing. Furthermore, average intensities of background areas near the observed particles were used to determine the course of bulk protein concentration over time.

Various conjectures exist on the cause of signal attenuation, which is regularly observed in confocal laser scanning microscopy. ${ }^{20-23}$ To account for these effects, irrespective of their origin, two normalization conditions were set. First, at time $t=0$ no protein is present within the adsorbent particle and, second, the particle is naturally assumed to be filled homogeneously under saturation conditions.

Consequently, the intraparticle profile data were normalized by subtraction of a second degree polynomial, which was fitted to the signal intensity profile at time $t=0$. Here, the choice of a second degree polynomial accounts for the nonlinearity of the observed aberration. The bulk phase concentration was 

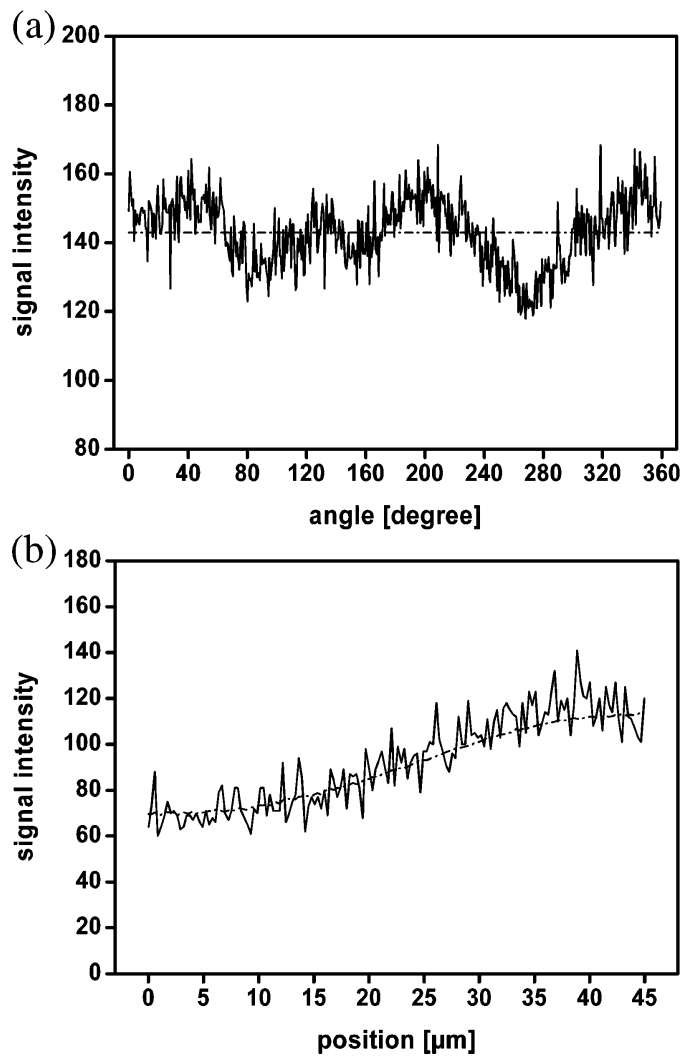

Figure 4. (a) Comparison of typical signal variations over the particle circumference at a fixed radius (solid line) and the respective average value (dash dotted line). (b) Comparison of a typical intensity profile over the particle radius at a fixed azimuth (solid line) and the respective result of the smoothing procedure (dash dotted line).

similarly normalized by subtraction of a constant. This normalization results in flat profiles with zero mean and consistent boundary condition at time $t=0$.

The intraparticle profile data were normalized a second time by division by a second degree polynomial, which was fitted to the signal intensity profile at a time when the particle was saturated. The resulting profile data are flat at saturation conditions and proportional to the protein concentration in the particle. The absolute values of the profiles are not relevant, as any constant factor cancels out of the model equations, provided the corresponding boundary condition $\hat{b}(t)$ is consistently normalized (see below). Consequently, all figures show relative concentrations $c(r, t) / c_{\max }$ throughout this paper.

The average bulk concentration near the examined particles was observed to be largely proportional to the average concentration at the outermost shell of the inside of these particles. This legitimates two assumptions for the given experimental conditions, which significantly simplify modeling and subsequent parameter estimation: First, the proportionality constant, which coincides with the phase ratio $\Phi$, was considered concentration independent, and second, the film mass transfer resistance was regarded irrelevant and, hence, neglected.

The concentration at the outermost shell of the particle inside, which is required as boundary condition for the diffusion model, is given by the product $\hat{b}(t)=\Phi b(t)$ of the phase ratio and the bulk phase concentration. This product is normalized by division by its maximum value, to establish consistency with the profile data. Note, that the phase ratio cancels out of the normalized data. The area for the determination of the bulk concentration profile was chosen as large as possible and preferably such as to surround the entire particle.
A disk with radius $4.35 \mu \mathrm{m}$ from the center of the particle was excluded from data analysis, due to low data availability for the azimuthal averaging procedure. Moreover, a ring with radius $4.35 \mu \mathrm{m}$ from the particle boundary was excluded from data analysis, because irregular signals were observed near the boundary, owing to imprecise particle edge detection. The resulting data were used for the estimation of intraparticle diffusion coefficients.

Spheric Diffusion Model. The intraparticle diffusion coefficient was determined from the experimental data by parameter estimation. A spheric diffusion model, with time variant boundary condition, was fitted to the data (eqs 1-3). This model requires knowledge of the protein concentration at the particle boundary, which, in contrast to the introduced pulse, does not resemble a sharp step function, to simulate the time course of the intraparticle protein concentration profile.

$$
\frac{\partial}{\partial t} c(r, t)=D_{\text {ip }}\left(\frac{1}{r^{2}} \frac{\partial}{\partial r}\left(r^{2} \frac{\partial}{\partial r} c(r, t)\right)\right)
$$

Here, $c$ denotes the intraparticle concentration, $t$ the time, $r$

$$
\begin{gathered}
c(r, 0)=0 \\
c(R, t)=\hat{b}(t)
\end{gathered}
$$

the radial position, $D_{\text {ip }}$ the intraparticle diffusion coefficient, $R$ the particle radius, and $\hat{b}$ the concentration at the inner particle boundary.

Equations 1-3 define a partial differential equation in time and space for the intraparticle protein concentration $c$, with initial value at time $t=0$ and boundary condition at radius $r=R$. This partial differential equation can be transformed to a system of ordinary differential equations by the method of lines: The partial derivatives of the concentration $c$ with respect to the radius $r$ were discretized at equidistant knots, which resemble the discrete structure of the experimental data. The resulting system of ordinary differential equations can be handled by any standard solver for stiff systems.

The system of differential equations was integrated using the MATLAB algorithm "ode15s". The resolution of the solution was around 160 radial knots and 180 frames in time, depending on the analyzed data set. Each individual model solution took approximately half a second. Here, all calculation times refer to a Pentium 4 system under Windows XP, which was clocked with $2 \mathrm{GHz}$.

Parameter Estimation. The estimation of the diffusion coefficient is an iterative process, which starts from an initial guess. The model was solved for a series of systematically varied diffusion coefficients for the complete data set at all time points and the corresponding simulation results were compared with the experimental data, to determine the best fit. The validity of the model and the consistency of the data were verified by the residual of the best fit, since the predicted concentration profiles were rather complex and the applied model has only one adjustable parameter. The error of the estimated coefficient was finally estimated by repetition of the entire procedure with different sets of experimental data for the same protein.

The MATLAB algorithm "fminbnd" was used to estimate the intraparticle diffusion coefficients. The differences between simulation results and experimental data were minimized in the sense of least squares. Two initial values were chosen more than an order of magnitude apart, to embrace the unknown value of the sought coefficient. The parameter estimation, within an absolute tolerance of $10^{-14}$, took about 20 iterations, resulting 
in an overall calculation time in the order of $10 \mathrm{~s}$, depending on the analyzed data set.

Stokes-Einstein Equation. In free solution and in the absence of interactions with other macromolecules, the temperature dependence of the diffusion coefficient can be written in the form of the Stokes-Einstein equation. ${ }^{24}$

$$
D=\frac{k_{\mathrm{b}} T}{6 \pi \eta R_{\mathrm{H}}}
$$

Here, $k_{\mathrm{b}}$ denotes Boltzmann's constant, $T$ the temperature in kelvin, $\eta$ the solution viscosity, and $R_{\mathrm{H}}$ the hydrodynamic radius. Equation 4 can be used to calculate the diffusion coefficient for a given temperature of, for instance, $20{ }^{\circ} \mathrm{C}$ from data given at other temperatures (eq 5).

$$
D_{20}=\frac{293.2}{T} \frac{\eta_{T}}{\eta_{20}} D_{T}
$$

Here, $D_{20}$ and $D_{\mathrm{T}}$ denote the diffusion coefficients at $20^{\circ} \mathrm{C}$ and $T$ and $\eta_{T}$ and $\eta_{20}$ the viscosity at $20^{\circ} \mathrm{C}$ and $T$. The diffusion coefficient $D$ is influenced by both the temperature and the viscosity of the solvent. Equation 5 is derived from two instances of eq 4, with different temperatures, by elimination of the hydrodynamic radius and solution of the resulting equation for the sought diffusion coefficient.

\section{Results and Discussion}

CLSM Measurements. The interpretation of data obtained when using CLSM as a tool for in situ determination of protein adsorption and transport behavior is highly dependent on the change of system parameters due to the covalent attachment of fluorescent labels on the protein surface.

Protein Size and Shape. The covalent attachment of a fluorescent dye like Cy5 increases the molecular weight by 792 Da. In comparison with the size of proteins the change in the diffusive behavior due to the increase in molecular weight is most likely negligible. A second assumption is that the attachment of a label will not significantly alter the structure of the respective protein. Both has indeed been demonstrated in a number of studies. 8,9

Matrix Interactions. When measuring intraparticle diffusion coefficients, it is necessary to rule out adsorption effects of the solute with the stationary phase. It has been shown that the attachment of fluorescent labels might lead to a distinct change of adsorptive behavior between the native and labeled protein. ${ }^{25}$ It is thus necessary to evaluate possible artifacts arising from the use of fluorescent labels or mode of detection when determining intraparticle diffusion coefficients. It is generally assumed that for many size-exclusion media especially at moderate ionic strength there exits negligible adsorptive interaction of proteins with the matrix. The conjugation of a fluorescent dye to a protein could lead to adsorptive interactions of the bioconjugate with the stationary phase. Figure 5 shows retention times of different fluorescent dyes and nitrate on Sepharose 6 FF in phosphate buffer (25 mM, pH 7.0, $200 \mathrm{mM} \mathrm{NaCl})$. Nitrate and Cy5 elute as a Gaussian peak with similar retention times, which is expected for a nonadsorbing substance. In contrast to that, both BODIPY FL and Atto-635 show increased retention times with respect to nitrate indicative of adsorptive interactions. Also in each case a second component is visible that could represent dimers of the dyes.

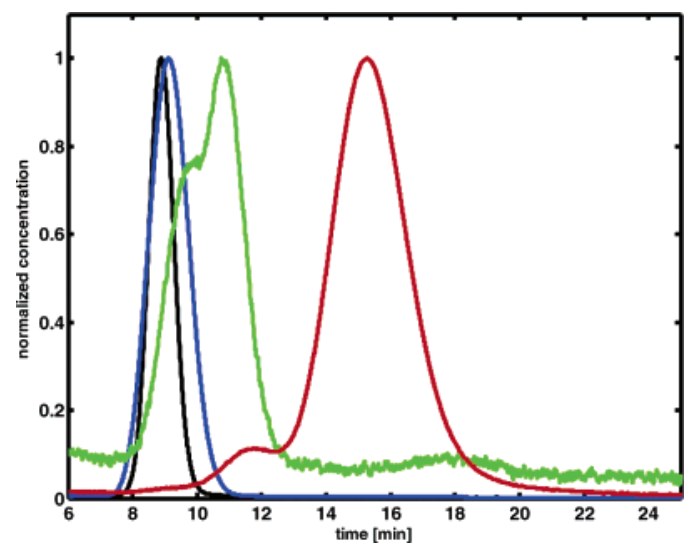

Figure 5. Normalized chromatograms of nitrate (black), Cy5 (blue), BODIPY FL (green), and Atto-635 (red): Tricorn 5/200 column with Sepharose $6 \mathrm{FF}$; flow rate, $0.2 \mathrm{~mL} / \mathrm{min}$; injection volume, $20 \mu \mathrm{L}$.

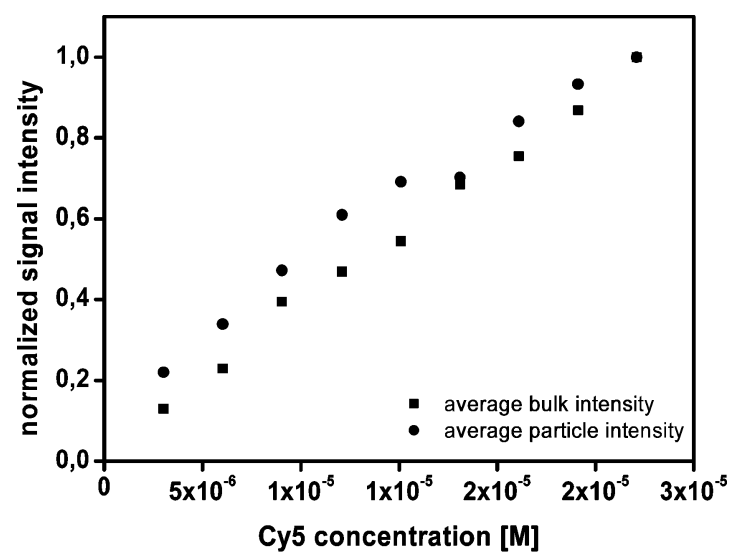

Figure 6. Normalized average fluorescence signal intensity for bulk (ם) and particle (-) phase, measured with CLSM and a 96-well plate at different $\mathrm{Cy} 5$ concentrations.

It is therefore important to choose an appropriate fluorescent dye for diffusion studies in porous media with $\mathrm{Cy} 5$ as a suitable conjugate in this study.

Signal Linearity. Figure 6 shows the linearity of the fluorescent signal measured in the bulk fluid phase and within a given particle. Signal linearity is given up to a dye concentration of at least $1 \times 10^{-5} \mathrm{M}$. The linear range for detection in this study is considerable higher than that obtained in earlier studies investigating the adsorption behavior of proteins on various stationary phases..$^{19,26,27}$ In these studies a low ratio of labeled to native protein molecule had to be applied in order to experimentally distinguish between labeled proteins in solution and accumulated on the solid phase. Due to the nonadsorbing nature of the underivatized stationary phase used in this study, only protein in the fluid phase will be detected and thus the ratio of labeled to unlabeled protein is increased to facilitate detection. It is worth mentioning that by altering the ratio of labeled to unlabeled protein in solution-for a given concentration of labeled species necessary for a linear signal-the protein solution can be set to different concentration values and thus resembles realistic conditions found during process chromatography.

Protein Concentration in the Bulk Phase. A necessary prerequisite for the computation of diffusion coefficients is the exact knowledge of the protein concentration in the fluid surrounding the stationary phase. Figure 7 shows the average protein concentration in the surrounding bulk phase over time as measured by CLSM. Although the protein sample is injected in the form of a step, the increase of concentration in the bulk 


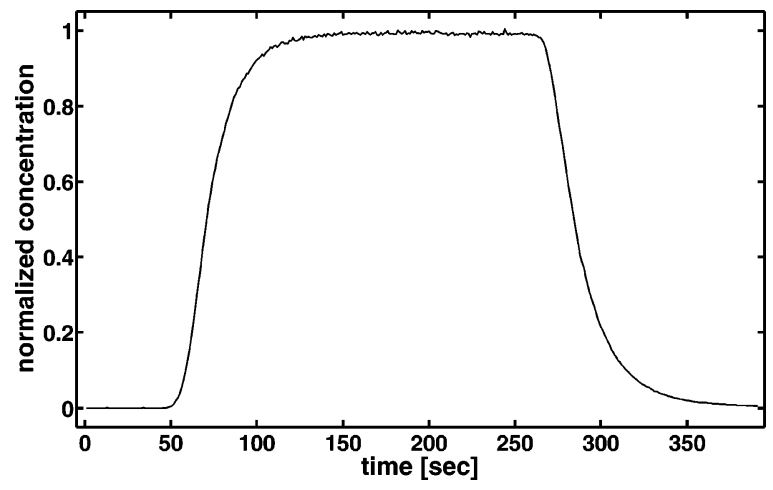

Figure 7. Protein concentration in the bulk phase near the analyzed particle over time for bovine serum albumin-Cy5 as determined via CLSM.

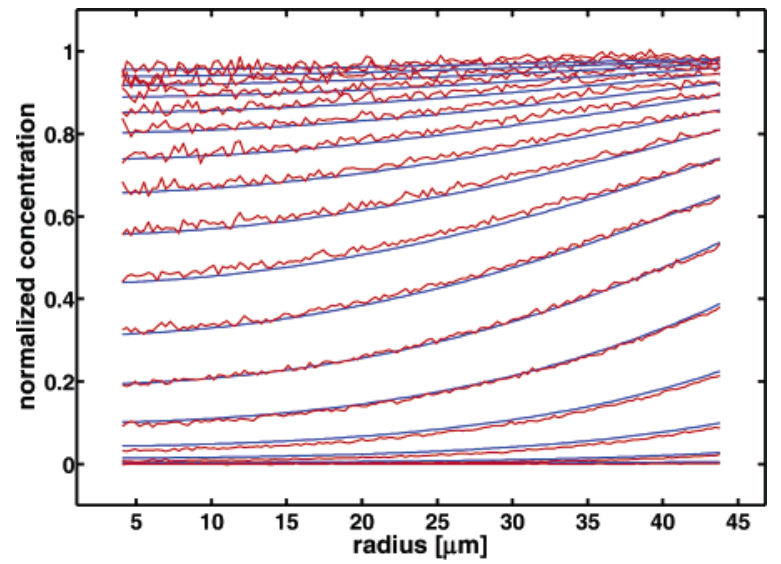

Figure 8. Measured (red) and simulated (blue) intraparticle protein concentration profiles at different times for diffusion of bovine serum albumin-Cy5 into the particle.

phase does not resemble a step function. Moreover, the wash out phase shows a strong tailing. The observed curvature is caused by dispersion of the sample. Under the assumption of an ideal step function the simulation results would not match the experimental data (see Figures S1 and S2 of the Supporting Information). Thus, the determination of the true concentration profile in the particle surrounding fluid phase over time is essential for a correct simulation of the experimental data.

\section{Computer Simulation}

The spheric diffusion model, which is used for the estimation of the intraparticle diffusion coefficient, has only one adjustable parameter. This parameter $D_{\text {ip }}$ is initially unknown and estimated from measurement data. Figures 8 and 9 show preprocessed concentration profiles from intraparticle measurements and simulation results from the spheric diffusion model at different times for one of the experiments. The simulations are based on the optimal diffusion parameter, which is previously determined by parameter estimation, and reproduce the experiments very well. A movie, which shows the time evolution of both experimental data and simulation results, is available as Supporting Information (Figure S3). The excellent match of simulation results with experimental data underlines the feasibility of the presented approach and indicates realistic results. An incorrect or incomplete model, with only one parameter, could most certainly not as accurately reproduce the dynamics of the entire diffusion process, as actually shown in Figures 8 and 9. The model passes the $\chi^{2}$ test with $90 \%$ confidence and better, even if the standard deviation of the averaged profile data is

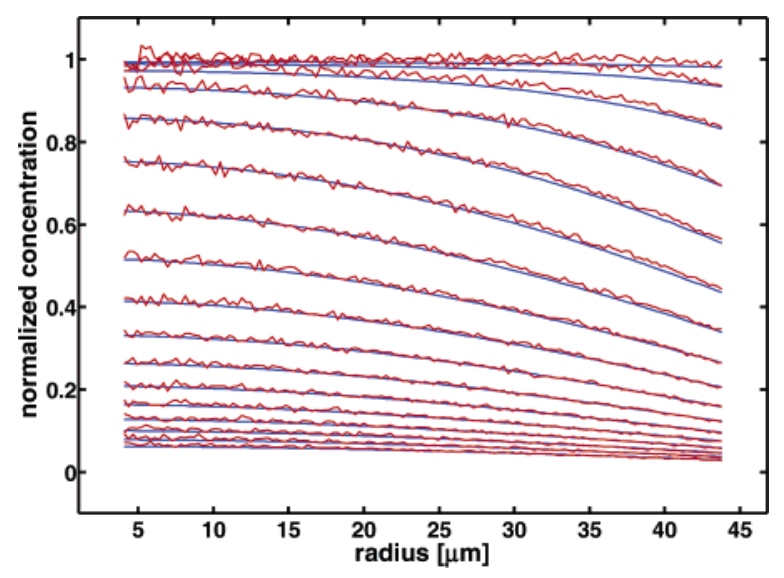

Figure 9. Measured (red) and simulated (blue) intraparticle protein concentration profiles at different times for diffusion of bovine serum albumin-Cy5 out of the particle.

estimated as low as $1 \%$ of the maximal value at saturation conditions.

Diffusion Coefficients. In this study, seven different proteins are analyzed covering a range of molecular weights from 14.3 to $160 \mathrm{kDa}$. Generally, very good agreement of model-based predictions and experimental data is observed. For each protein, the experiments are repeated three times, resulting in a standard deviation of less than $1.8 \%$ of the mean. This proves a good reproducibility of the presented approach. Table 1 lists the determined intraparticle diffusion coefficients supplemented by the corresponding standard deviations and the respective free diffusion coefficients for the seven analyzed proteins and the ratio of both values as well as the molecular weight of the respective proteins.

In Figure 10, the determined intraparticle diffusion coefficients are compared with results from the literature. The coefficients which are determined by IDA evidently lie well within the range of diffusion coefficients published in other studies, sharing the same order of magnitude. A deviation from the observed trend of the intraparticle diffusion coefficient is visible for lysozyme and chymotrypsinogen, which will be discussed below.

Boyer and $\mathrm{Hsu}^{3}$ studied the intraparticle diffusion of myoglobin, $\beta$-lactoglobulin, ovalbumin, hexokinase, immunoglobin $\mathrm{G}$, and catalase in Sepharose CL-6B at $4{ }^{\circ} \mathrm{C}$ with a $50 \mathrm{mM}$ phosphate buffer at $\mathrm{pH} 7.0$ with chromatographic pulse experiments. Equation 5, with $\eta_{4}$ and $\eta_{20}$ from Lide, ${ }^{29}$ is used to transfer these data to $20{ }^{\circ} \mathrm{C}$. The original diffusion coefficients presented by Boyer and $\mathrm{Hsu}$ represent effective diffusion coefficients. To account for the latter, we thus divided the respective effective diffusion coefficients by the respective effective porosities stated by the same authors.

Bosma and Wesselingh ${ }^{4}$ performed chromatographic pulse experiments with Q Sepharose FF as porous medium. The analyzed proteins were cytochrome $c$, lysozyme, ovalbumin, bovine serum albumin, and $\gamma$-globulin in a $1 \mathrm{M}$ acetate buffer at $\mathrm{pH}$ 4.4. A stage model, where the film diffusion was described as mass transfer through two films in series (i.e., liquid and particle film), was then fitted to the pulse experiments in order to obtain the intraparticle diffusion coefficients. The large deviation to other published coefficient values might thus be explained by the fact that their intraparticle diffusion coefficients resulted from a correlation with the particle film model as compared to using a particle model. Furthermore, the lower $D_{\text {ip }}$ can be explained by their use of positively charged particles (Q Sepharose FF), $1 \mathrm{M}$ acetate buffer, and different $\mathrm{pH}$. An 
TABLE 1: Intraparticle and Free Diffusion Coefficients Ratio of Both in Sepharose 6 FF As Determined by IDA

\begin{tabular}{|c|c|c|c|c|c|}
\hline protein & $D_{\mathrm{ip}}\left(\mathrm{m}^{2} / \mathrm{s}\right)$ & $\mathrm{SD}^{a}\left(\mathrm{~m}^{2} / \mathrm{s}\right)$ & $\mathrm{D}_{\mathrm{f}}^{b}\left(\mathrm{~m}^{2} / \mathrm{s}\right)$ & $D_{\mathrm{ip}} / D_{\mathrm{f}}$ & $\mathrm{MW}^{c}(\mathrm{Da})$ \\
\hline lysozyme & $6.07 \times 10^{-11}$ & $2.61 \times 10^{-13}$ & $1.15 \times 10^{-10}$ & 0.529 & 14300 \\
\hline$\alpha$-chymotrypsinogen A & $5.51 \times 10^{-11}$ & $2.41 \times 10^{-13}$ & $9.43 \times 10^{-11}$ & 0.584 & 25700 \\
\hline$\beta$-lactoglobulin ${ }^{d}$ & $4.86 \times 10^{-11}$ & $1.04 \times 10^{-13}$ & $8.84 \times 10^{-11}$ & 0.550 & 36500 \\
\hline ovalbumin & $3.81 \times 10^{-11}$ & $4.67 \times 10^{-13}$ & $8.32 \times 10^{-11}$ & 0.458 & 42800 \\
\hline bovine serum albumin & $2.45 \times 10^{-11}$ & $6.85 \times 10^{-13}$ & $6.15 \times 10^{-11}$ & 0.398 & 66400 \\
\hline ovotransferrin & $2.18 \times 10^{-11}$ & $9.59 \times 10^{-13}$ & $5.81 \times 10^{-11}$ & 0.375 & 75800 \\
\hline$\gamma$-globulin & $1.48 \times 10^{-11}$ & $7.50 \times 10^{-13}$ & $4.42 \times 10^{-11}$ & 0.335 & 160000 \\
\hline
\end{tabular}

${ }^{a}$ Standard deviations (SD) are estimated from three experiments. ${ }^{b}$ Measured with FCS. ${ }^{c}$ The molecular weight of the proteins is calculated from the amino acid sequence (www.uniprot.org). ${ }^{d} \beta$-Lactoglobulin is treated as a dimer. ${ }^{28}$

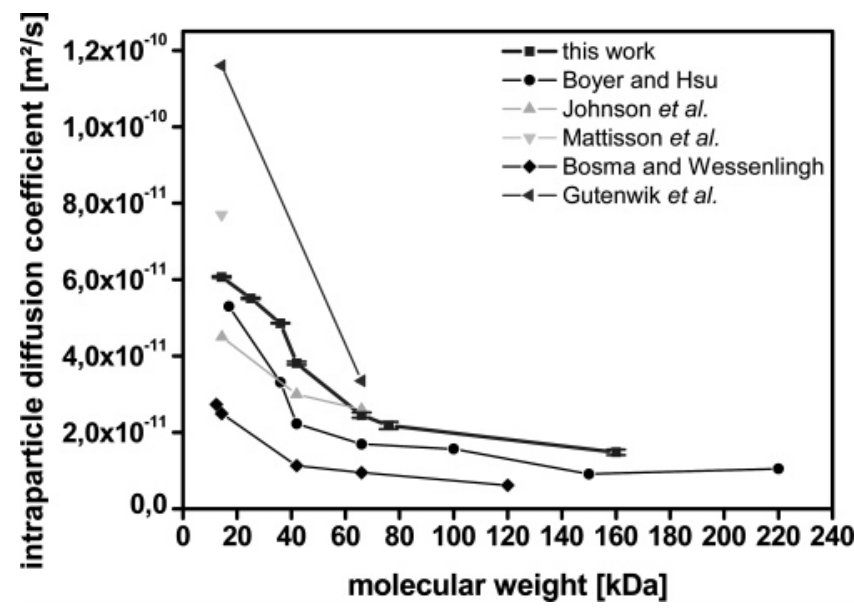

Figure 10. Intraparticle diffusion coefficients $D_{\text {ip }}$ over molecular weight. Results of IDA (ם) are compared with results of other approaches from literature.

increase in ionic strength with acetate ions may cause changes in the protein structure, which in turn could lower the diffusivity. Furthermore, Doherty and Benedek ${ }^{30}$ reported decreasing diffusivity as a result of increasing ionic strength. Interactions between proteins and ligands could lead to lower diffusivity in charged porous systems; however, Bosma and Wesselingh ${ }^{4}$ employed a high ionic strength, to screen for electrostatic interactions. Finally, the ligands potentially decrease the pore radius of the stationary phase in comparison to the underivatized media and, thus, lower the diffusivity.

Gutenwik et al. ${ }^{6}$ and Mattisson et al. ${ }^{12}$ did not analyze porous particles, but casted electrophoresis grade agarose of different concentrations with presumably lower tortuosities than Sepharose $6 \mathrm{FF}$, using either a stirred cell ${ }^{6}$ or interferometric methods. ${ }^{12}$ They chose bovine serum albumin ${ }^{6}$ and lysozyme ${ }^{6,12}$ as proteins. Results for pH 5.6 and $0.1 \mathrm{M} \mathrm{NaCl}$ from Gutenwik et al. ${ }^{6}$ and Mattisson et al. ${ }^{12}$ were taken for comparison.

Johnson et al. ${ }^{7}$ analyzed negatively charged SP Sepharose FF with the FRAP approach with bovine serum albumin, ovalbumin, and lactalbumin at an ionic strength of $1 \mathrm{M}$ and $\mathrm{pH}$ 7.1. Sepharose $6 \mathrm{FF}$, which is used in the present study, is the underivitized form of SP Sepharose FF and could, due to the absence of sulfopropyl ligands, have a larger pore radius. Furthermore, FRAP quantifies self-diffusion of proteins in porous media at equilibrium conditions, whereas IDA captures dynamic protein diffusion into and out of the matrix. Coffman et al. ${ }^{16}$ reported, that diffusivity is lower at higher protein concentration. The lower intraparticle diffusion coefficient of Johnson et al. ${ }^{7}$ could, hence, be explained by a smaller pore radius or higher protein concentration inside the particle.

In Table 1 , the intraparticle diffusion coefficients $D_{\text {ip }}$ are compared to the corresponding diffusion coefficients $D_{\mathrm{f}}$ in free solution. As expected, the coefficient $D_{\text {ip }}$ is generally lower than $D_{\mathrm{f}}$ and the ratio of $D_{\text {ip }}$ to $D_{\mathrm{f}}$ decreases with increasing molecular weight with the exception of lysozyme and chymotrypsinogen. This contradictory result is most likely attributable to adsorptive interactions between either lysozyme or chymotrypsinogen and Sepharose 6 FF which slows down the diffusion of the proteins. The agarose, which is used for chromatography, contains very small amounts of negativly charged groups. ${ }^{31}$ In contrast to the other proteins, which are analyzed in this study, both lysozyme and chymotrypsinogen carry a net positive charge at $\mathrm{pH} 7.0$, which could explain their unusual behavior.

\section{Conclusions}

The usage of underivatized stationary phase has the advantage of decoupling the diffusion processes from other secondary and multimodal interactions between the particle and the protein. It is generally hard to rule out all interactions between proteins and the solid interphase, but the experimental system chosen in this study should be very close to a pure hindered diffusion process without any adsorptive interactions. Further experiments could help in establishing general transport correlations for proteins in chromatographic stationary phases. This in turn can aid in decoupling diffusion processes from adsorption when modeling protein transport and adsorption in derivatized chromatographic materials.

Although hindered protein diffusion is investigated for a long time, there is still a lack of comparative data sets for several proteins under different solution conditions. Individual data from literature are not directly comparable, since they are generated by use of different experimental techniques. Direct intraparticle diffusion analysis (IDA) is designed for the fast and accurate quantification of protein diffusion in porous particles. IDA is a suitable tool for the comprehensive screening of various proteins and stationary phases. IDA essentially consists of two stages: First, intraparticle and bulk concentration profiles are measured over time, using confocal laser scanning microscopy and a custom designed microcolumn. Underivatized media are analyzed on a single particle level under moderate salt concentrations, to minimize interactions of proteins with the stationary phase. The second stage of IDA is a model-based data analysis and estimation of the intraparticle diffusion coefficient, using a spheric diffusion model with time variant boundary condition. The presented approach is successfully applied for the determination of intraparticle diffusion coefficients $D_{\text {ip }}$ of seven different proteins. Sepharose $6 \mathrm{FF}$ is used as stationary phase, whose derivatized forms have significant industrial relevance. For all analyzed proteins, the simulation of protein diffusion both in to and out of the particle matches excellently with the experimental data. The results compare well to results from literature, which are determined using a variety of different techniques. Further work will focus on the effect of solute concentration as well as ionic strength, on the $\mathrm{pH}$, and on the analysis of different porous media. Moreover, the approach is easily transferable to the study of multicomponent diffusion processes. IDA will be developed into a tool for transport 
analysis of large molecules in porous systems, which may yield not only more quantitative information but also a better understanding of the governing mechanisms. This is especially important in derivatized media where changes in the pore sizes and electrostatic or hydrophobic effects will influence the diffusion behavior. With the IDA approach the diffusion coefficient can be measured using the same media as in a purification step.

Finally existing theories on hindered diffusion in porous structures ${ }^{32}$ will be applied to the experimental results in order to gain a better mechanistic understanding of the particle size parameters that influences diffusion.

Acknowledgment. The authors greatly acknowledge the financial support of DFG (German Research Association). We thank Dr. Ingo Gregor from the Institute of Biological Signal Processing at the Research Center Juelich for the FCS measurements. We further thank Professor M.-R. Kula for her support and stimulating discussions.

Supporting Information Available: Movies showing experimental results for $\beta$-lactoglobulin-Cy5 and simulations with measured bulk concentration and assumed step function in bulk protein concentration. The information is available free of charge on the Internet at http://pubs.acs.org.

\section{References and Notes}

(1) Horstman, B. J.; Chase, H. A. Modeling the affinity adsorption of immunoglobulin-G to protein-A immobilized to agarose matrixes. Chem. Eng. Res. Des. 1989, 67, 243-254.

(2) Skidmore, G. L.; Horstman, B. J.; Chase, H. A. Modeling singlecomponent protein adsorption to the cation exchanger S Sepharose FF. $J$. Chromatogr. 1990, 498, 113-128.

(3) Boyer, P. M.; Hsu, J. T. Experimental Studies of Restricted Protein Diffusion in an Agarose Matrix. AIChE J. 1992, 38, 259-272.

(4) Bosma, J. C.; Wesselingh, J. A. Partitioning and diffusion of large molecules in fibrous structures. J. Chromatogr. B 2000, 743, 169-180.

(5) Davies, P. A. Determination of Diffusion Coefficients of Proteins in Beaded Agarose by Gel Filtration. J. Chromatogr. 1989, 483, 221-237.

(6) Gutenwik, J.; Nilsson, B.; Axelsson, A. Determination of protein diffusion coefficients in agarose gel with a diffusion cell. Biochem. Eng. J. 2004, 19, 1-7.

(7) Johnson, E. M.; Berk, D. A.; Jain, R. K.; Deen, W. M. Diffusion and partitioning of proteins in charged agarose gels. Biophys. J. 1995, 68, $1561-1568$

(8) Moussaoui, M.; Benlyas, M.; Wahl, P. Diffusion of proteins in the chromatographic gel ACA-34. J. Chromatogr. 1991, 558, 71-80.

(9) Moussaoui, M.; Benlyas, M.; Wahl, P. Diffusion of proteins in Sepharose Cl-B gels. J. Chromatogr. 1992, 591, 115-120.

(10) Pluen, A.; Netti, P. A.; Jain, R. K.; Berk, D. A. Diffusion of Macromolecules in Agarose Gels: Comparison of Linear and Globular Configurations. Biophys. J. 1999, 77, 542-552.

(11) Roger, P.; Mattisson, C.; Axelsson, A.; Zacchi, G. Use of Holographic Laser Interferometry to Study the Diffusion of Polymers in Gels. Biotechnol. Bioeng. 2000, 69, 654-663.
(12) Mattisson, C.; Roger, P.; Jonsson, B.; Axelsson, A.; Zacchi, G. Diffusion of lysozyme in gels and liquids A general approach for the determination of diffusion coefficients using holographic laser interferometry. J. Chromatogr., B 2000, 743, 151-167.

(13) Karlsson, D.; Zacchi, G.; Axelsson, A. Electronic Speckle Pattern Interferometry: A Tool for Determining Diffusion and Partition Coefficients for Proteins in Gels. Biotechnol. Prog. 2002, 18, 1423-1430.

(14) Gibbs, S. J.; Chu, A. S.; Lightfoot, E. N.; Root, T. W. Ovalbumin diffusion at low ionic strength. J. Phys. Chem. 1991, 95, 467-471.

(15) Gibbs, S. J.; Lightfoot, E. N.; Root, T. W. Protein Diffusion in Porous Gel Filtration Chromatography Media Studied by Pulsed Field Gradient NMR Spectroscopy. J. Phys. Chem. 1992, 96, 1458-1462.

(16) Coffman, J. L.; Lightfoot, E. N.; Root, T. W. Protein Diffusion in Porous Chromatographic Media Studied by Proton and Fluorine PFG-NMR. J. Phys. Chem. B 1997, 101, 2218-2223.

(17) DePhillips, P.; Lenhoff, A. M. Pore size distributions of cationexchange adsorbents determined by inverse size-exclusion chromatography. J. Chromatogr., A 2000, 883, 39-54.

(18) Böhmer, M. Pampaloni, F.; Wahl, M.; Rahn, H. J.; Erdmann, R. Enderlein, J. Advanced Time-Resolved Confocal Scanning Device For Ultrasensitive Fluorescence Detection. Rev. Sci. Instrum. 2001, 72, 41454152.

(19) Hubbuch, J.; Linden, T.; Knieps, E.; Thömmes, J.; Kula, M.-R. Dynamics of Protein Uptake Within the Adsorbent Particle During Packed Bed Chromatography. Biotechnol. Bioeng. 2002, 80, 359-368.

(20) Malmsten, M.; Xing, K. Z.; Ljunglof, A. Confocal microscopy studies of trypsin immobilization on porous glycidyl methacrylate beads. J. Colloid. Interface Sci. 1999, 220, 436-442.

(21) Heinemann, M.; Wagner, T.; Doumeche, B.; Ansorge-Schumacher, M.; Büchs, J. A new approach for the spatially resolved qualitative analysis of the protein distribution in hydrogel beads based on confocal laser scanning microscopy. Biotechnol. Lett. 2002, 24, 845-850.

(22) Heinemann, M.; Limper, U.; Büchs, J. New insights in the spatially resolved dynamic $\mathrm{pH}$ measurement in macroscopy large adsorbant particles by confocal laser scanning microscopy. J. Chromatogr., A 2004, 1024, 4553.

(23) Tallarek, U.; Rapp, E.; Sann, H.; Reichl, U.; Seidel-Morgenstern, A. Quantitative study of electrokinetic transport in porous media by confocal laser scanning microscopy. Langmuir 2003, 19, 4527-4531.

(24) Einstein, A. Über die von der molekularkinetischen Theorie der Wärme geforderte Bewegung von in ruhenden Flüssigkeiten suspendierten Teilchen. Ann. Phys. 1905, 17, 549.

(25) Teske, C. A.; Schroeder, M.; Simon, R.; Hubbuch, J. ProteinLabeling Effects in Confocal Laser Scanning Microscopy. J. Phys. Chem. B 2005, 109, 13811-13817.

(26) Ljunglöf, A.; Hjorth, R. Confocal Microscopy as a tool for studying protein adsorption to chromatographic matrixes. J. Chromatogr., A 1996, $743,75-83$

(27) Ljunglof, A.; Thömmes, J. Visualising intraparticle protein transport in porous adsorbents by confocal microscopy. J. Chromatogr., A 1998, 813, $387-395$.

(28) Brownlow, S.; Cabral, J. H. M.; Cooper, R.; Flower, D. R.; Yewdall, S. J.; Polikarpov, I.; North, A. C. T.; Sawyer, L. Bovine $\beta$-lactoglobulin at 1.8 A resolution-still an enigmatic lipocalin. Structure 1997, 5, 481-495.

(29) Handbook of Chemistry and Physics; Lide, D. R., Ed.; CRC Press: Boca Raton, FL, 2004.

(30) Doherty, P.; Benedek, G. B. The effect of electric charge on the diffusion of macromolecules. J. Chem. Phys. 1974, 61, 5426-5434.

(31) Duckworth, M.; Yaphe, W. The Structure of Agar. Carbohyd. Res. 1971, 16, 189-197.

(32) Amsden, B. Solute diffusion within hydrogels. Mechanisms and models. Macromolecules 1998, 31, 8382-8395. 